\title{
Chronic Traumatic Encephalopathy and Age of First Exposure To American-style Football
}

\author{
Alvaro Pascual-Leone, MD, $\mathrm{PhD}^{1}$ and Ross D. Zafonte, DO ${ }^{2}$ \\ ${ }^{1}$ Berenson-Allen Center for Noninvasive Brain Stimulation and Division for Cognitive Neurology, \\ Beth Israel Deaconess Medical Center; Department of Neurology, Harvard Medical School; \\ Boston MA \\ 2Physical Medicine and Rehabilitation, Brigham and Women's Hospital, Massachusetts General \\ Hospital, and Spaulding Rehabilitation Hospital; Harvard Medical School; Boston MA
}

In the present issue of Annals of Neurology, Alosco et al. [1] report important new findings regarding the relation between age of first exposure to American-style football and chronic traumatic encephalopathy (CTE). This is a very important study that contributes addresses a topic of great fundamental and clinical relevance. Alosco et al. [1] do not find a significant association between age of first exposure to tackle football and CTE pathology. However, they report a significant association between age of first exposure to tackle football and the age of onset of reported cognitive, behavioral and mood symptoms. These findings have great relevance regarding our understanding of CTE and its possible clinical correlates, as well as implications for the care of contact sports athletes.

According to a consensus statement from the National Institute of Neurological Disorders and Stroke and the National Institute of Biomedical Imaging and Bioengineering [2], CTE is a progressive neurodegenerative disease characterized by the brain deposition of pathogenic phosphorylated tau protein (P-tau). The etiology and pathophysiology of CTE remain unclear. Animal models reveal that deposition of pathogenic cis conformation of the phosphorylated Thr231-Pro motif of tau protein can be causally related to head trauma [3]. The pattern of P-tau deposition consistent with the human characteristics of CTE, and P-tau accumulation triggers a neurodegenerative cascade with neuronal cell death along functional brain networks [3]. However, in humans a causal link between repeated head trauma or traumatic brain injury (TBI) and CTE has not been established, and CTE-like pathology is also found in patients with temporal lobe epilepsy in absence of a history of TBI [4]. Thus, repetitive head trauma or TBI may be associated with CTE, but may not be the only cause.

Therefore, the pioneering work of the Boston University group is extremely important. They have previously reported pathological evidence of CTE at autopsy in the brains of 177 of

Corresponding author: A. Pascual-Leone, MD, PhD; Berenson-Allen Center for Non-invasive Brain Stimulation, Department of Neurology, Beth Israel Deaconess Medical Center, 330 Brookline Ave (KS-158), Boston, MA 02215, USA.

Potential Conflicts of Interest

Dr. Pascual-Leone serves on the scientific advisory boards for Nexstim, Neuronix, Starlab Neuroscience, Neuroelectrics, Constant Therapy, Cognito, and Neosync. Dr Zafonte serves on the Scientific Advisory Board of Myomo, Oxeia Biopharma, and ElMINDA. He also evaluates patients in the MGH Brain and Body-TRUST Program which is funded by the NFL Players Association. 
202 former American-style football players, including 110 of 111 former National Football League (NFL) players [5]. Even if these 110 brains were the only ones in the entire population of former NFL players to ever show CTE, the overall risk of the disease would still be substantial. Since 1960 there have been approximately 15,000 former NFL players, though exact numbers are hard to get given varying definitions of what qualifies as an NFL player. Even if no additional cases of CTE were to be found among former NFL players, the prevalence would still be about 7.5 in 1,000. This would be 7.5 higher than the standard of significant risk used by the Occupational Health and Safety (OSHA) Laws in the USA to regulate the safety of an industry or worksite [6]. CTE is a pathological and probably clinical health threat that demands a sober assessment of data and new scientific insights to enable evidence-based management decisions.

Many of the former athletes found to have CTE at autopsy, manifested mood disorders, headaches, cognitive and speech difficulties, suicidal ideation, and aggression during their lifetime. Nonetheless, it is uncertain whether the pathological diagnosis of CTE is obligatorily linked to clinical manifestations, and if so, what factors determine individual susceptibility and influence clinical phenotypes. The study by Alosco et al. [1] is particularly important in this context. They find a striking dissociation between objective measures of CTE pathological severity and subjective reports of clinical symptoms. Contrary to common assumptions, age of first exposure to tackle football was NOT associated with CTE pathological severity, but it did significantly influence reported age of onset of cognitive and behavioral/mood symptoms.

Alosco et al [1] suggest that exposure to tackle football earlier in life may reduce the brain's resilience to cope with neuropathology and thus lead to clinical manifestations earlier. This is a reasonable hypothesis that offers a plausible explanation to the findings predicated on the appealing, but unproven assumption, that CTE pathology is responsible for the clinical manifestations. However, it could be that the cognitive, behavioral and mood symptoms might not be caused - or at least might only be partially caused - by CTE pathology. For example, selection and recall bias may have affected the findings regarding clinical phenotype. Age of first exposure to tackle football and age of onset of cognitive and behavioral/mood symptoms were assessed by retrospective informant interviews. The informants were largely the same family members who donated the former NFL players' brains because they were concerned about the possibility of CTE. As Alosco et al. [1] discuss, informants may have been more likely to attribute symptoms to tackle football in those former players with more severe and earlier clinical manifestations. Recall bias may have been further exacerbated by the public discourse regarding the association between tackle football, CTE, and cognitive and behavioral disability. In addition, the complex context of class-action law suits and litigation regarding concussion settlement may have also contributed. The fact that Alosco et al. found the same relation between age of first exposure to tackle football and neurobehavioral symptoms in former players without CTE pathology, further supports the notion that cognitive, behavioral and mood disturbances may not be solely due to CTE. This would be in line with prior findings by Alosco et al. [7] in 214 former amateur and professional football players. In that study, they found no relation between age of first exposure to American-style football and performance in neuropsychological tests (Brief Test of Adult Cognition by Telephone), but a significant 
association with subjective self-reported measures of executive function and behavioral regulation.

If confirmed, the dissociation between CTE pathology and clinical symptoms is a very important finding with significant clinical implications. Presently, when confronted with cognitive or mood symptoms, former football players and contact sports athletes tend to assume their symptoms must be caused by CTE, a progressive and currently incurable condition. This could exacerbate perception of existing symptoms and greater disability. Clinically it may be warranted for former athletes to consider additional evaluation of treatable causes of their symptoms - e.g. obstructive sleep apnea, chronic pain, anxiety or depression.

Several important knowledge gaps regarding CTE remain. For example, the populations in which CTE has been described arguably represent an extreme of exposure to repeated head trauma and the significance of CTE for the general population remains unclear. Ultimately, we need to clarify whether CTE pathology is causally linked to clinical manifestations or merely associated with them. Emerging diagnostic tests in vivo, including serum, physiologic and imaging biomarkers, will help. Following youth athletes before, during and after their sporting careers could provide assessment of individual differences, cumulative effect of exposure, the contribution of associated risks, and the longer term consequences. As Alosco et al. [1] point out, clarifying the clinical correlates of CTE pathology requires longitudinal prospective studies with careful, serial evaluations, and the comprehensive consideration of genetic and epigenetic individual differences and multi-system interactions. The Football Players Health Study at Harvard University (https:// footballplayershealth.harvard.edu) is an important initiative in this regard. Until then, studies as the one by Alosco et al. are extremely valuable and, while they need to be interpreted with caution, they warrant public health consideration, and should lead to careful assessment of sports rules by the appropriate governing bodies.

\section{Acknowledgments}

Partly supported by the Football Players Health Study at Harvard University, and Harvard Catalyst | The Harvard Clinical and Translational Science Center (NCRR and the NCATS NIH, UL1 RR025758). The content is solely the responsibility of the authors and does not necessarily represent the official views of Harvard Catalyst, Harvard University and its affiliated academic healthcare centers, or the National Institutes of Health.

\section{References}

1. Alosco A, Mez J, Tripodis Y, et al. Age of first exposure to tackle football and chornic traumatic encephalopathy. Annals of Neurology. 2018

2. McKeeAnn C, et al. The First NINDS/NIBIB Consensus Meeting to Define Neu-ropathological Criteria for the Diagnosis of Chronic Traumatic Encephalopathy. 131 ACTA NEUROPATHOLOGY. 2016; 75

3. Kondo A, Shahpasand K, Mannix R, et al. Antibody against early driver of neuro-degeneration cis P-tau blocks brain injury and tauopathy. Nature. 2015; 523:431-6. [PubMed: 26176913]

4. Puvenna V, Engeler M, Banjara M, et al. Is phosphorylated tau unique to chronic traumatic encephalopathy? Phosphorylated tau in epileptic brain and chronic traumatic encephalopathy. Brain research. 2016; 1630:225-40. [PubMed: 26556772] 
5. Mez J, Daneshvar DH, Kiernan PT, et al. Clinicopathological Evaluation of Chronic Traumatic Encephalopathy in Players of American Football. JAMA. 2017 Jul 25; 318(4):360-370. DOI: 10.1001/jama.2017.8334 [PubMed: 28742910]

6. Finkel AM, Deubert CR, Lobel O, et al. The NFL as a Workplace: The Prospect of Applying Occupational Health and Safety Laws to Protect NFL Workers. 60 Ariz. L. Rev. 2018; 291

7. Alosco ML, Kasimis AB, Stamm JM, et al. Age of first exposure to American foot-ball and longterm neuropsychiatric and cognitive outcomes. Transl Psychiatry. 2017 Sep 19.7(9):e1236.doi: 10.1038/tp.2017.197 [PubMed: 28926003] 